\title{
POLYOSTOTIC FIBROUS DYSPLASIA
}

\author{
BY \\ R. H. VINES \\ From The Hospital for Sick Children, Great Ormond Street, London
}

(RECEIVED FOR PUBLICATION DECEMBER 11, 1951)

Polyostotic fibrous dysplasia is a disease that deserves to be better known for it is more frequent than is generally appreciated. Von Recklinghausen's (1891) description of what appear to be two examples of this disease together with one of hyperparathyroidism seems to have set a precedent for many fruitless parathyroid explorations. This has occurred despite Hunter and Turnbull's (1931) differentiation of 'osteitis fibrosa in multiple foci' from 'osteitis fibrosa cystica generalisata'.

Interest in the condition and a recognition of the frequent association with other features was aroused by Albright, Butler, Hampton and Smith (1937) in a paper describing a syndrome of disseminated osteitis fibrosa, skin pigmentation, and, in females, sexual precocity. Despite earlier descriptions by McCune and Bruch (1937) and Weil (1922) this syndrome has often been called Albright's syndrome.

Lichtenstein (1938) in a review and pathological report suggested the term polyostotic fibrous dysplasia. In his view the bony lesion is the one essential feature of the disease.

Three patients with this disease have been seen in The Hospital for Sick Children, since 1938. One was reported by Warrick (1949) and is referred to by Jolly (1951). This was a boy who showed skin pigmentation, involvement of the skull and probably two ribs, and sexual and skeletal precocity. His parents and his four siblings showed no skin pigmentation. The other two are reported in this paper for the first time.

\section{Case Reports}

Case 1. J.F., a boy aged 6 when first admitted to this hospital on June 30, 1946, was referred to Dr. Pearson on account of bone cysts. These had been discovered when he sustained a pathological fracture of the right humerus in October, 1945. Further fractures of the right humerus and right radius had occurred during treatment in a plaster cast, but all had healed well. Before these events he had always been well. He was re-admitted on several further occasions with pathological fractures.

He had three siblings, one of whom, an elder sister, showed two café an lait patches on the right thigh.
His mother had a small, faint café au lait patch over the left cubital fossa. Her only sister showed no pigmentation. The boy's father showed none and it was said that the father's nine siblings showed none. The boy's maternal grandmother had a small, faint café au lait patch on the left arm. His maternal grandfather was said to be free of these spots, but had a sister who had a small, dark cafe au lait patch on the abdomen; there were five other siblings in this family. The patient's mother had no x-ray evidence of bone disease in the left arm and his sister with pigmentation had no lesions of the skeleton on $x$-ray.

Examination in January, 1948, showed a thin, alert boy with a bony swelling on the upper and outer side of the right forearm, a palpably enlarged right humerus and slight limitation of supination on that side. The tonsils and tonsillar glands were enlarged. No abnormality was found on clinical examination of other bones, heart, lungs, abdomen, genitalia, nervous system or neck. A small café au lait patch was present on the lower right abdominal wall.

Radiographs showed diffuse involvement of the humeri (Fig. 1) which were expanded, the cortex thinned in places, and the normal structure replaced by translucent and 'ground glass' areas. Similar cystic appearances were seen in both radii, several metacarpals and phalanges in both hands, in the eleventh right rib and the right femur and tibia. The skull was normal. The bone age remained normal. There was no generalized osteoporosis. Some progression of the lesions occurred during the period of observation. An intravenous pyelogram showed no abnormality.

A biopsy from the right humerus showed replacement of the marrow spaces by fibrous tissue. 
Examination of the urine showed absence of protein and Bence-Jones protein on several occasions.

Biochemical investigations gave a blood calcium level of $10 \cdot 4,10 \cdot 2,9 \cdot 7,9.4 \mathrm{mg}$. per $100 \mathrm{ml}$; inorganic serum phosphorus, $3 \cdot 6,3 \cdot 8,3 \cdot 4,3 \cdot 3 \mathrm{mg}$. per $100 \mathrm{ml}$; serum alkaline phosphatase, $27 \cdot 5,32 \cdot 7,37 \cdot 8,45 \cdot 8$ units. Two four-day calcium balance periods showed a positive calcium balance of $0.44 \mathrm{~g}$. a day and $0.34 \mathrm{~g}$. a day respectively.

The blood urea, plasma proteins, blood count and blood sedimentation rate were normal.

Case 2. X.C., a 13-year-old boy of Greek parentage and Egyptian domicile, was sent from Cairo by Dr. Cardiacos to Dr. Lightwood and admitted on June 21, 1951 , with bilateral knock knee. This had been noted when the boy was 6 years of age and had increased rapidly in the last three years. Areas of skin pigmentation had been present at birth. Puberty had not occurred unduly early.

Birth weight was $10 \mathrm{lb}$. and his early development normal. He had been jaundiced for a month when 6 years old, had Giardia dysentery when 7 , and a transient left pleural effusion when aged 10.

The parents were healthy. His only sibling, a 3-yearold girl, had a small brown spot on the right thigh seen in a photograph. The paternal grandfather was reliably reported to have had brown pigmented areas of skin on the back and right thigh.

Examination showed an intelligent boy of average height and a little over average weight for his age (Figs. 2 and 3). The complexion was dark and on the face, neck, right thorax, buttocks and thighs there were extensive areas of brown pigmentation with irregular edges. A little straight pubic hair was present but no axillary or facial hair. The genitalia were normal for his age. The skull showed marked frontal and parietal bossing

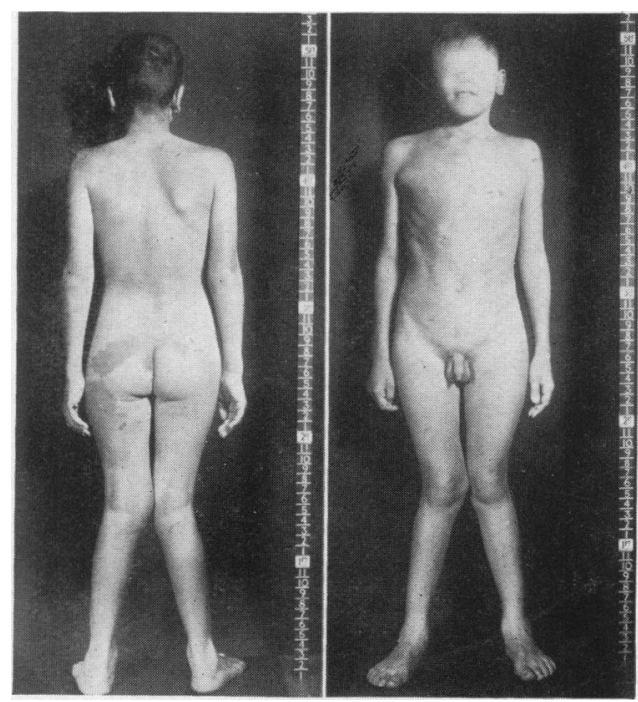

Figs. 2. Figs. 2 AND 3. Case 2. giving the 'hot cross bun' effect. There was facial asymmetry with prominence of the left forehead. The nasal bridge was flat and wide, the interpupillary

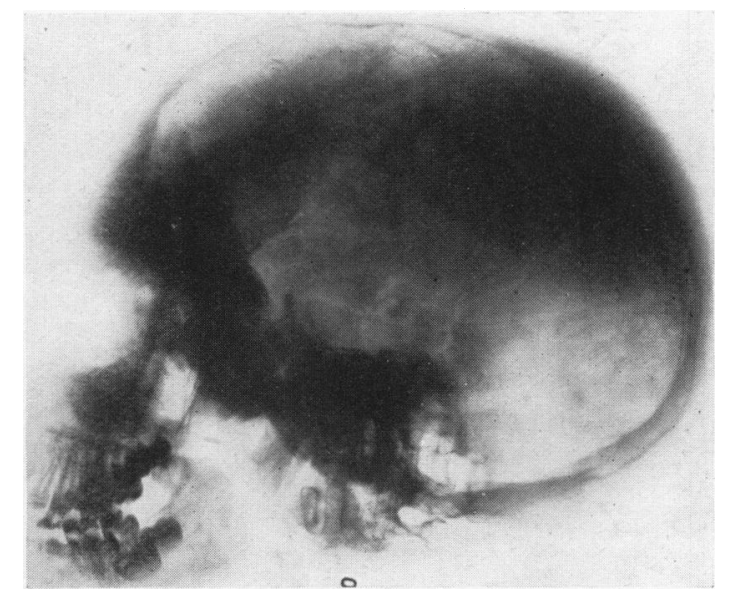

FIG. 4.-Radiograph of skull of Case 2.

distance being $171 \mathrm{~mm}$. The upper limbs appeared normal apart from marked hyperextensibility of the metacarpophalangeal joints and an ability to do 'double-jointed' tricks by subluxating the right humerus at the shoulder joint. A mild lower dorsal kyphoscoliosis was present. The lower limbs showed bilateral genu valgum with seven inches intermalleolar separation. The thyroid isthmus was palpable. Detailed examination of vision and hearing only revealed some peripheral contraction of the visual fields, especially on the left with no other abnormality, nor was any abnormality found in the nervous system, heart, lungs or abdomen.

Radiographs (Figs. 4 and 5) showed that the bone age was within normal limits. There were marked thickening and patchy sclerosis of the base of the skull, the maxillae, the frontal bones and in the parietal regions. The femora showed large translucent areas and in places there was expansion of the shaft and thinning of the cortex. Similar changes were present in all bones except the clavicles, carpus, some phalanges, most ribs, some vertebrae and several tarsal bones.

A skin biopsy showed that the epidermis was normal apart from excessive melanin pigmentation of the rete Malpighi. Van Gieson's stain was taken up abnormally by the deeper areas of dermal collagen, and there was mild perivascular infiltration with small round cells and histiocytes, some of the latter containing melanin granules. The vessels and elastic network of the dermis were normal.

Bone biopsy showed the typical features of osteitis fibrosa, that is, residual bone trabeculae embedded in dense fibrous tissue.

Biochemical investigations gave an inorganic serum phosphorus level of $3.1 \mathrm{mg}$. per $100 \mathrm{ml}$.; blood calcium of $11.2 \mathrm{mg}$. per $100 \mathrm{ml}$; ; alkaline serum phosphatase of 84 units. Blood urea, chlorides and bicarbonate were normal. 
Blood Wasserman and Kahn reactions and the Mantoux test $1: 100$ were negative. No protein or Bence-Jones protein was found in two specimens of urine. Amino-acid excretion was normal.

As it appeared likely that the bone disease was still active, stapling over the lower femoral epiphyses was carried out to ameliorate the knock knee. Later when activity will presumably be less, osteotomies may be required.

\section{Discussion}

Polyostotic fibrous dysplasia is a disease of childhood, the lesions becoming arrested in adult life. The sexes are equally affected. Attention is usually called to it by deformities, by pathological fracture, by pain, or less frequently, by the associated endocrine changes.

The Bony Lesion. One or many bones may be affected. Schlumberger (1946) considers it impossible to relate monostotic instances of fibrous dysplasia to the polyostotic form and believes the former follows bone injury. That some cases where one bone alone is affected do belong with the polyostotic group is perhaps supported by the concurrence of monostotic disease and pigmentation in the case of Dockerty, Ghormley, Kennedy and Pugh (1945), Jaffe's (1946) two cases, Russell and

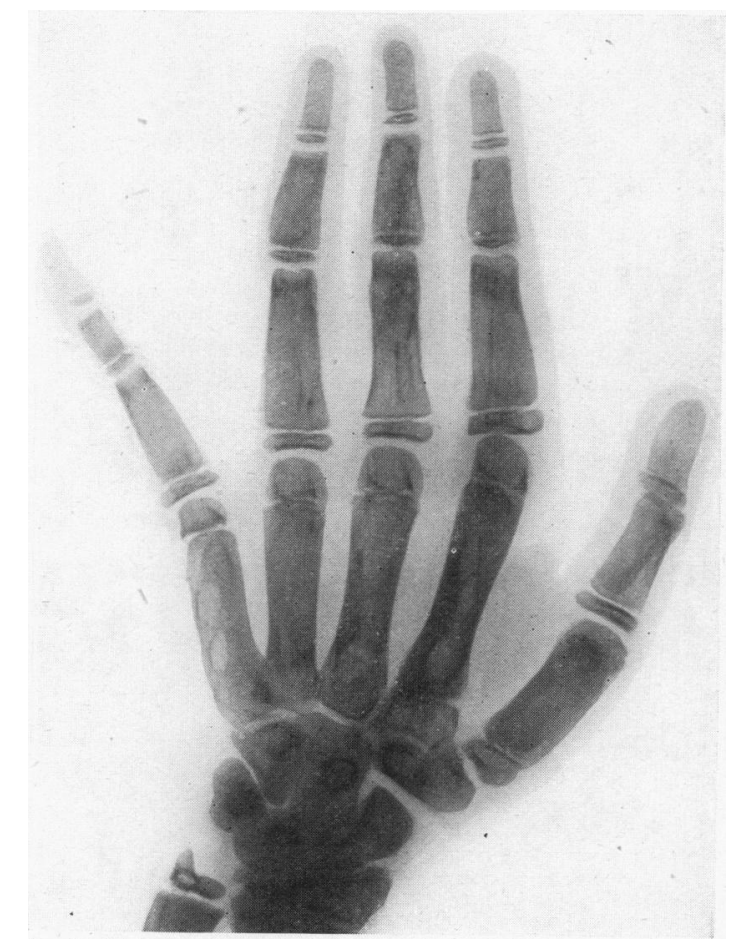

FIG. 5.-Radiograph of hand of Case 2. (Both hands showed a similar picture.)
Chandler's (1950) two cases and the two cases of Strassburger, Garber and Hallock (1951). The disease tends to affect the bones developed from one limb bud or to be unilateral. The proximal long bones and the base of the skull are most often affected. Epiphyses are rarely involved and then only after fusion.

Falconer, Cope and Robb-Smith (1942) think that the histology suggests 'the primary change is a marrow fibrosis with secondary absorption of the laminated trabeculae and then a new formation of fibre bone'.

Additional features are the frequent presence of cartilage islets, occasionally of small cystic spaces and of small collections of xanthoma cells and rarely of osteoclastomata (Dockerty et al., 1945). Lichtenstein (1938) considers the presence of cartilage islets in the marrow fibrosis pathognomonic. Schlumberger (1946) has brought evidence to show that it is not and that it may be seen as part of the reparative process in bone after injury. Osteogenic sarcoma occurring in this disease has been reported by Coley and Stewart (1945, two examples), by Jaffe (1946) and by Snapper (quoted by Albright, 1947).

Radiologically the marrow fibrosis shows in thinning and expansion of the cortex of bones. Bending occurs and the 'shepherd's crook' deformity of the femur is typical. Normal bone pattern is lost, a 'ground glass' appearance replacing it together with dense trabeculae giving a cystic appearance. At the base of the skull involvement shows itself as dense sclerosis and overgrowth which may result in compression of cranial nerves.

Skin Pigmentation. Café au lait patches are seen in the majority of cases. There is some tendency for them to occur on the more affected side. Pigmentation has been reported on the buccal mucosa. Albright has repeatedly stressed the irregular margin of these areas and considers it a useful contrast with the smooth, regular-edged café au lait patches of neurofibromatosis. Histologically it is due to excessive melanin deposition, chiefly in the basal layer of the epidermis.

Endocrine Changes. The most frequent of these is skeletal precocity giving rapid early growth and eventual dwarfing. Less often there is sexual precocity which is common in females but rare in males (Lange, 1938; Falconer et al., 1942; Warrick, 1949, two cases). The sexual precocity is of the true or 'cerebral' type. Thyroid enlargement is not uncommon and several examples of hyperthyroidism are reported. Acromegaloid features were present in the two patients of Falconer et al. (1942) and in that of Peck and Sage (1944), and 
gynaecomastia has been seen by Moehlig and Schreiber (1940) and by Albright.

Chemistry. Calcium and phosphorus levels in the blood and calcium balances are normal. The only abnormality of blood chemistry is the frequent elevation of the alkaline serum phosphatase. In the urine Bence-Jones protein was found in the patient of Murray, Kirkpatrick and Forrai (1946) and in one of Albright's patients.

Aetiology. General opinion now inclines to consider this disease to be of congenital origin. Helfet (1940) and Uehlinger (1940) believed it might be due to chronic hyperparathyroidism. Bremer (1941) produced fibrous lesions in bone by giving oestrogens to experimental animals and thought this effect was produced through the parathyroids. Further evidence to support these ideas has not been brought forward and the often striking asymmetry of the lesions is a real difficulty in accepting them.

Four examples have been reported following neonatal jaundice (McCune and Bruch, 1937; Summerfeldt and Brown, 1939; Braid, 1939, two cases). Braid suggested that the condition might result from early liver damage, but there has been no more evidence to support this.

There has been no evidence that heredity plays any part. Hirsch (1929) reported three siblings with 'generalized hyperplastic malacia' and a history of 'spasms' in childhood. Particularly as he states that 'the cancellous structure is everywhere normal', I am unconvinced that these were examples of polyostotic fibrous dysplasia as Warrick (1949) suggests. However, in Cases 1 and 2 reported here there is a family history of skin pigmentation. The significance of this cannot be judged without knowing the incidence of such pigmentation in the population. To determine this 85 adults in the general medical wards of a London hospital and 15 children over the age of 5 were examined, in all 50 females and 50 males. A café au lait spot was defined as of developmental origin, faint to dark brown colour, being neither raised, palpable nor altering skin texture, and of at least $1 \mathrm{~cm}$. diameter. Five females showed them and seven males. Having shown such a high incidence of café au latt spots in the population as $12 \%$, the significance of the two family histories of pigmentation is doubtful.

Café au lait patches without other abnormality have been regarded by some as the forme fruste of neurofibromatosis. Taking this as a starting point Thannhauser (1944) has endeavoured to link neurofibromatosis with polyostotic fibrous dysplasia. Albright (1947) has defended the separateness of these entities. It would be of interest to determine whether the thickening and club-like expansion of the finest skin-nerves seen in the café au lait patches of neurofibromatosis (Stalmann, quoted by Thannhauser) is present in polyostotic fibrous dysplasia. There are no reports of this having been done.

Any explanation of the pathogenesis must account for the involvement of tissues from two germ layers present in three systems, and for the tendency to be unilateral. Albright and Reifenstein (1948) consider the lesion to be a disseminated neurological one, the sexual precocity being due to a hypothalamic lesion. Thannhauser's suggestion that the hypothalamus might be affected by overgrowth of bone at the base of the skull is difficult to maintain as not all examples with endocrine changes have shown skull involvement, radiologically at least.

Lichtenstein (1938) considers the bony lesion to be due to 'perverted activity of the specific bone-forming mesenchyme' and likens it to a hamartoma.

In an incomplete search of the literature, but covering some 140 cases with involvement of more than one bone, I have tried to discover the frequency of congenital abnormalities in this disease. If they were much increased there would be good evidence in support of a congenital origin.

Ten cases were found having associated congenital anomalies, namely: (1) mental retardation (Snapper and Parisel, 1933); (2) mental retardation, and at necropsy diminution in size of one mamillary body and an extra nucleus in the adjacent tissue (Albright, Butler, Hampton and Smith, 1937); (3) coarctation of the aorta and rudimentary left kidney (Coleman, 1939); (4) acyanotic congenital heart disease (Dockerty et al., 1945); (5) congenital arteriovenous fistulae of left arm and leg (Stauffer, Arbuckle and Aegerter, 1941); (6) vascular and pigmented naevi (cited by Jaffe, 1946); (7) Meckel's diverticulum and patent foramen ovale (Sternberg and Joseph, 1942); (8) pseudoxanthoma elasticum (Upjohn, 1951); (9) osteopoikilosis (Osgood, 1946); and (10) multiple pigmented naevi (Behrend, 1945).

In assessing the significance of this group the factor of selection must be considered because of the more thorough examination such people undergo. Selection may also have occurred in that those showing congenital abnormalities are more likely to be reported. No comparable figures for the incidence of congenital anomalies are available,

\footnotetext{
* A case with heart disease reported by Murray et. al., (1946) and the single cases with neurological lesions of Albright, Scoville and Sulkowitch (1938) and of Dockerty, et al. (1945) have not been included because of the possibility of non-congenital causation. Summerfeldt and Brown's (1939) case with an I.Q. of 70 must be included in the lower normal range.
} 
the $2 \%$ figure commonly accepted being the figure found in surveys of infants in the first month of life. Of the ten instances listed only Nos. 4, 5 and 6 and 10 are likely to have been included in such a survey. That an increased incidence of congenital anomalies exists in this disease has, therefore, not been proven.

Differential Diagnosis. The following have to be considered.

HYPERPARATHYROIDISM. This may be differentiated by the pains and weakness of the patient, the generalized osteoporosis, high serum calcium and negative calcium balance.

Neurofibromatosis. Papillomata, neurofibromata and few bony lesions tending to be located about the knee joint are shown.

PageT's Disease. Paget's disease occurs later in life and is a difficulty only when the skull alone is involved. Cortical thickening of bone occurs.

Ollier's Disease. This is recognized by the shortening and thickening of affected bones and by the frequent involvement of epiphyses.

Eosinophilic Granuloma and Lipoid ReticuLOSIS. These conditions usually show more sharply punched-out lesions and differentiation may be difficult without a biopsy.

Melorheostosis. Melorheostosis is differentiated by the presence of irregular cortical projections and the involvement of epiphyses.

Treatment and Prognosis. The expectation of life is usually not altered. In adult life progression of the lesions very rarely occurs though deformities and fractures may further complicate them. Fractures heal well. Treatment consists of the correction of deformities and the care of fractures. Though often unsuccessful, curettage and bone grafting sometimes give very good results.

\section{Summary}

The three instances of polyostotic fibrous dysplasia occurring at this hospital are reviewed and two of these are published for the first time. Both these new cases belong to families in which several members have café au lait spots. To determine the significance of this, 100 people were examined and 12 found to have one or more café au lait spots.

In view of this high incidence the significance of the family histories remains doubtful.

The condition is reviewed and current ideas of pathology and aetiology are discussed.

Ten cases with associated congenital abnormalities have been found in the literature. That this represents a high incidence of association has been shown to be unproven.

I wish to express my thanks to Dr. R. Lightwood for permission to publish his case and for his helpful criticism and to Dr. Cedric Carter for advice in the preparation of this paper.

\section{REFERENCES}

Albright, F., Butler, A. M., Hampton, A. O. and Smith, P. (1937). New Engl. J. Med., 216, 727.

Scoville, B. and Sulkowitch, H. W. (1938). Endocrinology, 22, 411 . (1947). J. clin. Endocr., 7, 307. and Reifenstein, E. C. (1948). "The Parathyroid Glands and Metabolic Bone Disease,' p. 263. Baltimore.

Behrend, A. (1945). Ann. Surg., 121, 245.

Braid, F. (1939). Archives of Disease in Childhood, 14, 181

Bremer, J. L. (1941). Arch. Path., Chicago, 32, 200.

Coleman, M. (1939). Brit. J. Surg., 26, 705.

Coley, B. L. and Stewart, F. W. (1945). Ann. Surg., 121, 872

Coley, B. L. and Stewart, F. W. (1945). A. Ann. Kennedy, R. L. J. and Pugh D. G. (1945). Arch. intern. Med., 75, 357.

Falconer, M. A., Cope, C. L. and Robb-Smith, A. H. T. (1942), Quart. J. Med., N.S. 11, 121.

Helfet, A. J. (1940).' Brit. J. Surg., 27, 651.

Hirsch, I. S. (1929). Radiology, 13, 44.

Hunter, D. and Turnbull, H. M. (1931). Brit. J. Surg., 19, 203.

Jaffe, H. L. (1946). Bull. N.Y. Acad. Med., 22, 588.

Jolly, H. (1951). Proc. roy. Soc. Med., 44, 459.

Lange, K. (1938). Zbl. Chir., 65, 2368.

Lichtenstein, L. (1938). Arch. Surg., 36, 874.

McCune, D. J. and Bruch, H. (1937). Amer. J. Dis. Child., 54, 806. Moehlig, R. C. and Schreiber, F. (1940). Amer. J. Roetgenol.,

Murray, R. C., Kirkpatrick, H. J. R. and Forrai, E. (1946). Brit. J. Surg., 34, 48.

Osgood, E. C. (1946). Amer. J. Roentgenol., 56, 174.

Peck, F. B. and Sage, C. V. (1944). Amer. J. med. Sci., 208, 35.

Recklinghausen, F, von (1891). In 'Festscrift. Rudolf Virchow zu seinem 71. Geburtstage.' Berlin.

Russell, L. W. and Chandler, F. A. (1950). J. Bone Jt Surg., 32A, $32 \dot{3}$.

Schlumberger, H. G. (1946). Milit., Surg., 99, 504.

Snapper, I. and Parisel, C. (1933). Quart. J. Med., N.S. 2, 407.

Stauffer, H. M., Arbuckle, R. K. and Aegerter, E. E. (1941). J. Bone Jt Surg., 23, 323.

Sternberg, W. H. and Joseph, V. (1942). Amer. J. Dis. Child., 63, 748 .

Strassburger, P., Garber, C. Z. and Hallock, H. (1951). J. Bone Jt. Surg., 33A, 407.

Summerfeldt, P. and Brown, A. (1939). Amer. J. Dis. Child., 57, 90.

Thannhauser, S. J. (1944). Medicine, Baltimore, 23, 105.

Uehlinger, E. (1940). Virchows Arch., 306, 255.

Upjohn, C. (1951). Proc. roy. Soc. Med., 44, 294

Warrick, C. K. (1949). J. Bone Jt Surg., 31B, 175.

Weil, -. (1922). Klin. Wschr., 1, 2114. 